\title{
RESEÑAS
}

\section{PEDAGOGÍA DE PROYECTOS, INTERCULTURALIDAD Y DINÁMICA SOCIAL: LE TRIANGLE DE L'ECUMEUR ${ }^{1}$}

\author{
ANNIE COÜEDEL* \\ NICOLE BLONDEAU* * \\ TATIANA KALENTIEVA***
}

\begin{abstract}
Todo proyecto educativo, ya sea individual o colectivo, expresa necesariamente una postura ideológica, sobretodo si se lo considera apolítico. En efecto, implica ciertas elecciones, opciones, la traducción de una visión del mundo y, por ende, de un proyecto de sociedad. Es necesario comprender que el objetivo de un proyecto educativo no es imponer de modo alguno convicciones ideológicas, sino de manifestar explícitamente las opciones sobre las cuales se fundamenta. En este sentido, lo pedagógico es inseparable de lo político.
\end{abstract}

Jacques Ardoino (Educación y política)

\section{INTRODUCCIÓN}

El proyecto fundador de Vincennes era político y las formas que adoptó la enseñanza derivaron, en gran parte, de las opciones políticas iniciales. Reflexionar hoy sobre las formas actuales de la práctica pedagógica implica necesariamente un retorno a lo político (Berger y Courtois, 1992). La pedagogía que se presenta aquí es una metodología de hecho, intercultural, con raíces en un determinado contexto -el de Paris 8, Vincennes en Saint Denis-, llevada a cabo por actores cuya intervención social, fuera del espacio cerrado del curso, se inscribe en una dimensión decididamente política.

Esta pedagogía - pedagogía de la acción y en relación con la sociedad- se sustenta en un dispositivo de investigación-acción en el que la relación estudiantes/estudiantes, estudiantes/profesores va a provocar la emergencia de proyectos y una dinámica social, tanto dentro como fuera de la Universidad.

Este dispositivo de investigación-acción sobre los factores que entran en juego en la adquisición de un idioma, en un principio concebido para un público de estudiantes extranjeros inscritos en lá universidad, se vincula con las investigaciones nacidas en Estados Unidos en el seno del pensamiento pragmático de los años 1915-1920.

En cierto modo, puede considerarse que la pedagogía de proyectos es una respuesta a las crisis del área educativa: fracaso escolar, burocratización, mayor rigidez del sistema educativo. En el centro del dispositivo se encuentra el sujeto, su inserción social y sus posibilidades de intervención: es el lenguaje concebido en su ejercicio cívico, como herramienta de intervención social, que une las diferentes concepciones (progresistas 1) de la pedagogía de proyectos.

\footnotetext{
${ }^{1}$ X Congreso Mundial de profesores de francés: Modernidad, Diversidad, Solidaridad organizado por la FIPF (Federación Internacional de Profesores de Francés), París 17-21de julio de 2000.

* Docente investigativa, Université de Paris 8 .

** Docente investigativa, Université de Paris 8.

*** Docente investigativa, Université des Langues d'Irkoutsk. annie.couedel@wanadoo.fr
} 
Los proyectos son colectivos y constituyen iniciativas inéditas para afrontar la realidad social. El objetivo es intervenir en los dispositivos sociales que se pretende transformar. Para que haya cambio debe haber una confrontación entre concepciones divergentes, incluso enfrentamiento. Por lo tanto, la interculturalidad está en el centro del proyecto, ya que los valores dominantes de los dispositivos sociales dados se enfrentan, se oponen, a otros valores.

\section{LAS CARACTERÍ́STICAS DE LOS PROYECTOS}

\subsection{Los proyectos som collectivos}

El grupo tiene una dimensión política dado que, a través del mismo, por el mismo, se aprenden y se ponen en práctica las reglas de moral social.

$\mathrm{Al}$ iniciarse el semestre universitario, cuando comienzan los talleres de concepción y realización de proyectos, los profesores enuncian un cierto número de principios que los estudiantes deben respetar: escuchar al otro, responsabilidad individual y colectiva, asiduidad y puntualidad, principios comunes a toda situación de enseñanza/aprendizaje.

En el mejor de los casos, los estudiantes recibirán dichos principios con respeto y atención. Tratarán de ponerlos en práctica, porque del cumplimiento de esas reglas depende, en parte, la validación/aprobación de la materia.

En los talleres, los principios son sometidos a la prueba del sentido que les dio origen: cuando un grupo está realizando un proyecto en común, el ausentismo, la falta de atención a la palabra del otro y el no respetar los compromisos pueden poner en peligro el logro del proyecto.

Poco a poco, los estudiantes se apropian de las reglas, negocian su modo de intervención, establecen parámetros para poder trabajar juntos. Entonces, las reglas dejan de ser fórmulas mágicas para ser valores democráticos que estructuran un trabajo en conjunto y garantizan que pueda llevarse a cabo.

El grupo supone una concepción diferente en la relación con la formación. Se aprende con los pares, en una elaboración común. Se aprende en conjunto, midiéndose con los otros, enfrentándose con los otros, chocando con maneras de pensar y representaciones del mundo que a veces pueden desestabilizar las que tenemos incorporadas. Dado que es único, el proyecto no puede acomodarse a modelos preexistentes. Por el contrario, va a movilizar la imaginación de cada uno y la participación igualitaria, aunque diferencial, de sus promotores.

El sistema pedagógico actual todavía funciona, en gran parte, con base en la comparación de los conocimientos y la competencia entre las personas, lo cual dificulta cualquier posibilidad de intervención colectiva, atomizando a los individuos en una lucha solitaria por ganarse un lugar. Por el contrario, en el grupo-proyecto, las capacidades y conocimientos de todos serán necesarias y funcionarán en sinergia. Cada uno aportará lo que pueda, lo que es en un determinado momento de su historia, progresando con los otros a medida que evoluciona el proyecto.

Corresponde, entonces, preguntarse qué lugar tiene un extranjero en el grupo. El dominio inseguro del idioma no será visto como un obstáculo, dado que no es el idioma lo que está en juego, sino la construcción de un objeto social en el que participa, como individuo, con los mismos derechos que los otros. Progresivamente, ayudado por sus pares y en confrontación permanente con el entorno, aprenderá la lengua, y la competencia social que necesariamente la acompaña, en el transcurso de una actividad o una práctica.

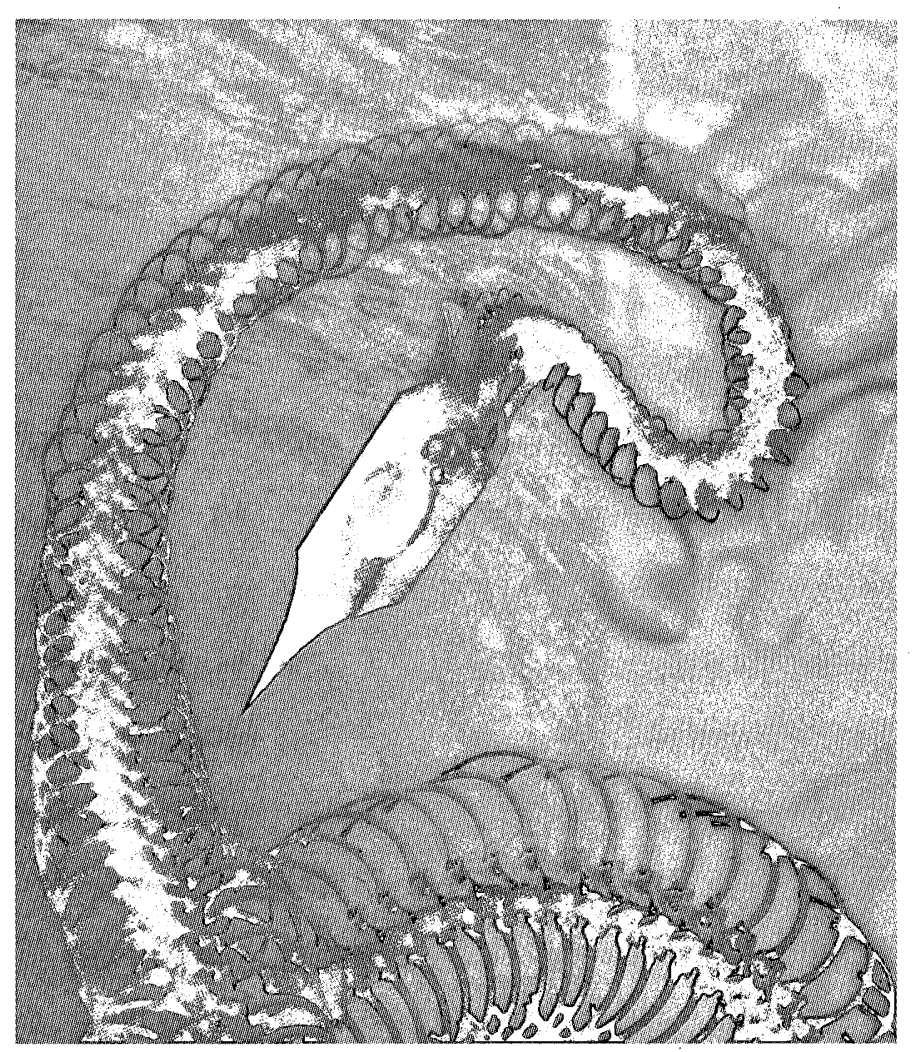


El grupo es un grupo-sujeto. De su cohesión, de la solidaridad entre sus miembros, depende la eficacia de la intervención en el exterior. No se puede actuar solo frente a una situación dada, si se quiere transformarla. Por lo tanto, el enfrentamiento con el exterior es colectivo y la inserción social individual se realiza gracias a lo colectivo.

\subsection{Los proyectos están relacionados com la sociedlad}

Los interrogantes que se plantean son los siguientes: "¿Qué universidad queremos? ¿Para qué sociedad?".

Los estudiantes de Paris 8 vienen a la universidad para estudiar, obtener diplomas y a menudo traen proyectos individuales que, al nò encontrar un lugar de expresión, medios de producción o una infraestructura, nunca se realizan. Por otra parte, una de las razones de los fracasos en el primer ciclo de las carreras universitarias se debe a la falta de comprensión de la organización pedagógica, a la ausencia de puntos de referencia claros en la institución:

La inserción del proyecto en la realidad social necesariamente lleva a los estudiantes a descubrir cómo funciona, quiénes son los actores, qué es lo que está en juego en una elección u otra. En una entrevista reciente Guy Berger dijo: "El proyecto se concibe como un modelo organizador que va a estructurar las relaciones con el exterior".

A partir del momento en que un proyecto toma forma, necesariamente hay negociaciones, confrontaciones con los diferentes estamentos administrativos. También, y sobre todo, se produce el descubrimiento y la percepción de grupos más o menos informales, cuyas posturas - a menudo ideológicas, pero jamás reivindicadas como tales-influencian la toma de decisiones. Los promotores de los proyectos aprenderán a identificar las relaciones de fuerza, los códigos y las normas que genera la institución, antes de poner en funcionamiento las estrategias de intervención que obligarán al sistema, en el mejor de los casos, a cooperar, o por lo menos a reaccionar. De todos modos, comprender cómo funciona una institución es comprender cómo funcionan todas las otras.

La actitud crítica respecto al entorno genera un proceso de distanciamiento y cuestionamiento de la sociedad. Aquí cabe hacer referencia al con- cepto de "concientización" de Paulo Freire, para quien la educación no puede pensarse independientemente del poder que la constituye, ni separada de la realidad concreta en la que se inserta. Para Freire, todo aprendizaje debe estar estrechamente asociado a la situación concreta que vive el alumno. El punto de partida consiste en admitir que la libertad y la crítica son derechos esenciales de la vida humana.

El grupo de trabajo y de intercambios, o "círculo de cultura", tiene como objetivo principal el estudio del lenguaje en el contexto de una práctica social libre y crítica. El hombre podrá transformar la realidad, su realidad, a través de la educación como acto político y la concientización. Freire opone su punto de vista a lo que denomina educación bancaria, es decir, aquella que se limita a depositar un saber ya constituido. Para él, toda educación debe ser dialógica, basada en los conocimientos y la práctica de los alumnos y fuertemente arraigada en el contexto cultural; una pedagogía de la libertad implica la adopción de actitudes democráticas, en el marco de acciones compartidas por todos: profesores y alumnos ${ }^{2}$.

\subsection{Los proyectos son creadores}

"Según se piense en términos de 'programa' o de 'proyecto' los modelos son diferentes" (Berger y Courtois, 1992). Si se considera la etimología, programa significa "lo que está escrito de antemano". Por lo tanto, se trata de un modelo de planificación, de previsiones, en un sistema cerrado que se construye con base en una progresión y etapas preconcebidas. Ya está escrito lo que va a ocurrir y las acciones están previstas para lograr un resultado predeterminado. El programa segmenta el tiempo, lo divide en unidades capitalizables que, una vez acumuladas, constituirán la totalidad de su contenido. Fascinado por los contenidos y la rentabilidad, el programa tiene muy poco en cuenta al sujeto, que debe adaptarse para no ser marginado.

En cuanto al proyecto, obliga a tener en cuenta todos los parámetros de la situación y las múltiples variables. No está definido de antemano y puede transformarse en función de las circunstancias, de lo que ocurra sin que esté previsto. Por lo tanto, los promotores van a desarrollar estrategias

\footnotetext{
2 Una pedagogía de proyectos puede ser tan coercitiva como una pedagogía basada en la relación tradicional profesor-estudiante, si la iniciativa de los proyectos no la tienen los estudiantes.
} 
inéditas, a inventar respuestas adaptadas para responder a lo imprevisto, a lo accidental, a la sorpresa. El proyecto se inscribe en la complejidad del mundo real. "Es la invención cotidiana" (Berger y Courtois, 1992).

Además, se sirve de múltiples conocimientos. No se lo puede reducir a una sola disciplina, sino que se nutre de conocimientos provenientes de diferentes disciplinas. Para la elaboración colectiva se activarán las diversas capacidades de los participantes. De este modo, se rompe el encierro disciplinario, la separación de los conocimientos, el individualismo.

La elaboración del proyecto y los logros dependen de personas libres y pensantes "que tienen algo que decir". Por lo tanto, si bien el grupoproyecto ve positivamente la emergencia de un líder que cataliza los deseos de cada uno y dinamiza los compromisos, la imposición de un jefe autocrático, de un pequeño tirano en potencia se convierte en un hecho problemático. El jefe se inscribe en la programación, la previsión. Estructura el tiempo en función de la percepción que tiene del proyecto y elimina las sugerencias que no coincidan con sus ideas. Reducidos al estado de simples ejecutantes, los otros participantes tienen que elegir entre la retirada, en cuyo caso el proyecto fracasa, o su reubicación como sujetos, portadores de ideas, valores y compromiso. Se trata de un verdadero aprendizaje de la democracia.

\section{LA INTERCULTURALIDAD ES UN ELEMENTO CENTRAL DEL PROYECTO}

Para nosotros, lo que se ha convenido en denominar interculturalidad es uno de los elementos de la heterogeneidad social en general y del grupo de asistentes a los talleres de pedagogía de proyectos. Los participantes son franceses y extranjeros, provenientes de cinco continentes, de orígenes sociales diversos, con edades comprendidas entre los 18 y 40 años, incluso 50; estudiantes de primer ciclo, pero también de maestría o de tesis, de las más variadas disciplinas: letras, artes, informática, filosofía, ciencias políticas, etc.

Esta heterogeneidad, lejos de ser un freno, se vive como un elemento motor para el aprendizaje. Crea una dinámica en las interacciones, multiplica los intercambios, provoca conflictos cuya regulación rara vez se hace hablando, pero sí en la realización del proyecto.
En efecto, no es el conocimiento que se adquiere de una diferencia denominada "cultural" lo que permite comprender realmente qué está en juego. Saber que ciertas formaciones culturales tienen una percepción del tiempo que E. T. Hall califica de policroma puede no ser más que un análisis interesante hasta el momento en que, confrontados a las personas en un contexto institucional, viviremos las consecuencias de dicha filosofía.

Ahora bien, si todos desean que el proyecto profesional tenga éxito, estaremos obligados a entrar en un proceso de negociación en el cual ni uno, ni otro de los interlocutores pierda terreno, ni tiene la impresión de haber abandonado lo que pertenece a su identidad sin obtener nada a cambio.

Los intercambios que se producen en los trabajos de grupo llevan la marca de la pertenencia cultural de sus locutores, sus prejuicios, sus interrogantes y, a veces, su agresividad respecto de la identidad perturbadora del otro. No obstante, los tropiezos en la comunicación no depénden solamente de lo cultural, sino también de lo social: posición que se ocupa, capital cultural, mayor o menor prestigio adjudicado a la propia cultura y a la del otro.

En el marco de un proyecto elaborado en común, los actores descubren cómo son percibidos, a través de la forma de organizar lo cotidiano, de interpretar las situaciones, de desarrollar estrategias, de abordar los problemas. En este proceso de elaboración común se trata de permitir que cada uno tome conciencia de que no existe un comportamiento cultural universal, que cada uno llega con un pasado, una historia que participa de la historia de un país y de una sociedad en relación con otras historias y otras sociedades.

La interculturalidad implica un avance de uno mismo hacia el otro, del otro hacia uno, un cambio en sus representaciones, una transformación que sólo puede elaborarse en un proceso común de confrontación de la realidad con otras realidades. Esta aceptación no puede darse si no es en el marco de una empresa realizada colectivamente, iniciada por sus actores y tomando un lugar y un sentido en sus vidas: en un proyecto construido en común.

Esta dinámica de puesta en funcionamiento de proyectos comunes es la que permite la creación de un lazo social entre personas de diferentes nacionalidades, lenguas y orígenes sociales. El papel 
del profesor cambia radicalmente y el proyecto puede entenderse como el ejercicio de un vínculo que describiremos como no autoritario, no piramidal y no institucional.

Precisamente gracias a este intercambio y a esta libertad compartida se constituirán espontáneamente las redes. El deseo de permanecer en contacto, de continuar la aventura intelectual y afectiva, que naturalmente llevará al deseo de reencontrarse en el país del otro durante un tiempo más o menos largo, para llevar a término y construir otros proyectos. Esta pedagogía supone un desplazamiento permanente de lo que está en juego, de las energías, de las perspectivas para conservar la dinámica. A menudo esto da a los dispositivos un aspecto rizomático a través del tiempo. El proyecto denominado Le Triangle de l'Ecumeur es un buen ejemplo.

\section{EL PROY ECTO: LE TRIANGLE DE L'ECUMEUR}

Este proyecto se inició con la realización de una revista del mismo nombre que vinculaba los talleres de concepción y realización de proyectos de Annie Couedel, de la Universidad Paris 8, con los estudiantes y la profesora Tatiana Kalentieva, de Irkutsk, en Rusia, proyecto al cual se unieron Guy Feve y sus estudiantes de la Universidad Francesa del Pacífico, en la Polinesia francesa.

El proyecto se amplió rápidamente, dado que la revista se extendió a los estudiantes y profesores de West Georgia en Estados Unidos, de Torreón en México y de Bogotá, en Colombia. De este modo, la naturaleza del proyecto fue cambiando paulatinamente y desde entonces dio lugar a encuentros interuniversitarios en diferentes partes del mundo. Parecería que la rapidez con la que los estudiantes se inscriben en la cultura del país de los amigos-actores de un mismo proyecto, en parte depende de la marcha del proyecto: el proyecto es el hilo conductor de una red que desafía las fronteras. Esto es lo que nos ha demostrado la experiencia de Le Triangle de l'Ecumeur.

¿Por qué Le Triangle de l'Ecumeur? Como bien lo dice Jacques Ardoino en su obra Education et politique (1977, p. 233) "Ya no es posible concebir nuestro aparato pedagógico independientemente de su contexto político" [...] "Hoy lo político desborda los límites de la ciudad, de la ciudad-estado y de la confrontación. Implica al conjunto del globo terrestre. Lo político es planetario", precisando, una vez más, que para él lo político "no es una técnica para acceder al poder o para mantener el poder tanto en el interior como en el exterior, sino una reflexión sobre los fines globales de la sociedad y sobre los modos de realización de ese proyecto".

\subsection{El encuentro de Le Triangle de l'Ecumeurr en París 8}

En el verano de 1997, 50 estudiantes y profesores franceses y extranjeros se encontraron en París y en Saint Denis en el marco del Encuentro Intercultural de Le Triangle de l'Ecumeur. Los estudiantes rusos provenían de Irkutsk (Siberia), los estudiantes colombianos de la Universidad Francisco José de Caldas de Bogotá (Colombia), los norteamericanos de West Georgia University (cerca de Atlanta, Georgia), los estudiantes de la Universidad Paris 8, franceses y extranjeros, eran también miembros del Centro Cultural de.Vincennes en Saint Denis.

Organizado conjuntamente por el Centro y el Departamento Comunicación/Francés Lengua Extranjera (FLE), el encuentro mencionado fue el tercero. El primero tuvo lugar en la Universidad Francesa del Pacífico (Papeete, Thaití) y el segundo en Irkutsk (Rusia), en el Instituto Universitario de Lenguas. El encuentro siguiente se llevó a cabo en Atlanta (Estados Unidos).

Estos encuentros internacionales, quizá los únicos de este tipo, no son solamente interculturales, sino también interlingüísticos (cuatro idiomas o más), interinstitucionales e interdisciplinarios (dado que los participantes provienen de diversas disciplinas: psicología, sociología, artes plásticas, cine, antropología, comunicación, lingüística). El tema elegido fue: la ciudad: espacio/tiempo.

El objetivo de este encuentro, como también de los precedentes, es de poner en contacto durante un mes, en sesión intensiva, a profesores y estudiantes de diferentes horizontes culturales y científicos, para llevar a cabo una investigaciónacción sobre los procesos de adquisición de una lengua y los elementos que intervienen en la comunicación intercultural, en el contexto actual de la globalización.

Además, se trata de experimentar, al mismo tiempo, en diversos contextos, la metodología de la pedagogía de proyectos, a fin de evaluar si es 
pertinente para llevar a cabo una investigación de este tipo.

\subsection{Los proyectos}

Desde su llegada a la universidad, los estudiantes constituyeron grupos utilizando diversos apoyos. En cada grupo había estudiantes de todas las nacionalidades presentes.

- Un taller-teatro trabajó sobre las diferencias culturales a través del tiempo y el espacio.

- Un grupo de música creó la letra y la música de varias canciones.

- Un grupo de expresión corporal representó varias situaciones de la vida cotidiana (desfases culturales).

- Otros estudiantes prefirieron interesarse en el poder de atracción mágica que pudo ejercer París a través de los años. Este grupo realizó un dossier ilustrado y comentado maravillosamente.

- Otro equipo se consagró enteramente a la publicación de un nuevo número de la revista $L e$ Triangle de l'Ecumeur, utilizando, además, los recursos de los cuadernos de bitácora de los participantes y el talento de los dibujantes y diseñadores del grupo. La revista pudo distribuirse a las tres de la mañana, el último día del encuentro. iUn buen ejemplo de inversión!

- Debe mencionarse también la presencia de un taller de cocina internacional y otro sobre el gesto y la identidad nacional.

- Se realizó un video sobre el desarrollo del encuentro, que será presentado en diferentes coloquios.

- Puede decirse que durante las tres semanas, más que hablar sobre la adopción de una metodología, lo que hubo fue una adaptación a la pedagogía mencionada por parte de los estudiantes y profesores extranjeros. Teniendo en cuenta las diferencias de los contextos institucionales representados, podría decirse que esta forma adaptada, pero respetada en sus principios fundamentales, es la apropiada para los intereses comunes.

Estos encuentros marcan de manera profunda a los participantes, inmersos en una "caldera mágica" donde se mezclan las fronteras entre los idiomas de origen, las disciplinas y los niveles de estudios. Le Triangle de l'Ecumeur provoca la creación de redes de intercambio que siguen funcionando más allá de los encuentros puntuales, fenómeno rápidamente incontrolable.

El acuerdo es institucional y los participantes lo firmaron al final de la reunión a fin de mantener el intercambio interuniversitario y realizar las tareas que se comprometieron a llevar a cabo: crear una red de investigadores, escribir una página en Internet, organizar conjuntamente seminarios y coloquios, intercambiar artículos y publicaciones sobre los temas: pedagogía de proyectos, aspectos interdisciplinarios e interculturalidad.

Nuestros amigos y colegas extranjeros han analizado más detalladamente, en sus informes, las diferencias culturales (antes y después del encuentro) sobre la representación del tiempo y del espacio, desde el punto de vista del individuo y del grupo.

Estas reuniones "gitanas", en las que el viaje llama al viaje en una confrontación con el tiempo y el espacio del otro, quizá nos brinden, como escribió Levi-Strauss: "uno de los últimos medios para no perder el alma" . Estos encuentros son, también, una voluntad de resistencia a la globalización.

\section{BIBLIOGRAFÍA}

ARDOINO, Jacques, (1977), Education et politique, París, Gauthier-Villars.

BERGER, G., y Courtois M., (1992), "Pédagogie et politique", La lettre de Paris 8, París, abril-mayo.

OURY, Ferdinand, (1982), Vers une pédagogie institutionelle, París, Maspéro.

SYLVIE Montazeau, (1997), "L'espace, le temps, la ville" [Informe del Encuentro], Universidad Paris VIII, París, octubre-noviembre.

\footnotetext{
${ }^{3}$ Philippe Meirieu, en su obra Apprendre, oui mais comment, habla de la mediación a través del proyecto y del ritual. Advierte sobre el peligro de la "relación pedagógica", la relación dual profesor-estudiante, que puede ser un factor de regresión, abrir la puerta a "identificaciones masivas de tipo caníbal, a regresiones inexplicables, a la confusión, a la mezcla de cuerpos, a la locura" (citado en Ferdinand Oury, 1982). Recuerda que "la más ancestral de las mediaciones, la que los hombres vieron enseguida que podía protegerlos contra el deambular y la emoción, es, sin duda, el ritual. Al imponer una organización del espacio y del tiempo, al asignar lugares, al codificar gestos y palabras, regula la vida colectiva, garantiza la seguridad de cada uno y define las fronteras de su acción [...]". Para Meirieu: "si no se puede dejar de lado la relación pedagógica [...] es importante administrar, hasta donde se pueda, la distribución y los efectos, introduciendo las mediaciones necesarias (...)".
} 
INSTRUCCIONES

A LOS AUTORES

\section{PARA $\llbracket A$ PRESENTACIÓN DE DOCUMENTOS}

El Comité Editorial de la Revista ENUNCIACIÓN, con fundamento en los requisitos establecidos para la Indexación de Publicaciones Seriadas Científicas y Tecnológicas Colombianas, PUBLINDEX, informa a sus colaboradores que ha definido los siguientes criterios para la evaluación, selección y publicación de artículos.

ołLa Revista Enunciación tiene una periodicidad anual.

- Los artículos que conforman la Revista corresponden a resultados de investigación, en consecuencia, deben exhibir coherencia conceptual, profundidad en el dominio de la problemática abordada así como estar escritos en un estilo claro, ágil y estructurado de acuerdo con la naturaleza del texto.

- La extensión máxima del artículo será de 20 cuartillas, formato Word, letra 12, tipo Arial, interlineado doble (excluyendo ilustraciones y cuadros).

- Además del texto impreso, los artículos deberán estar acompañados de medio magnético.

- Junto con el documento, deberá incluirse un abstract en lengua española y lengua inglesa (máximo de 150 palabras) y las palabras claves del artículo.

- La identificación del autor o autores del artículo debe incluir nombres y apellidos, títulos académicos, cargo actual, adscripción institucional y correo electrónico.

- Es necesario identificar la cobertura temática del artículo y adecuar los títulos y subtítulos teniendo en cuenta su brevedad y sentido.

- Las notas deben colocarse al final del texto, registrando su numeración en el cuerpo del artículo. Para las referencias bibliográficas, se sugiere adoptar el modelo de la APA.

- Teniendo en cuenta los criterios de clasificación establecido por COLCIENCIAS, "las revistas científicas y tecnológicas están orientadas esencialmente a la comunicación y discusión de resultados de investigación y desarrollo tecnológico. En la evaluación del contenido presente en las revistas, elemento determinante para establecer la calidad, se tendrá en cuenta los siguientes tipos de artículos, los cuales constituyen el conocimiento certificado, es decir, evaluado por pares:

a. Artículos de investigación científica y de desarrollo tecnológico: documentos que presentan de manera detallada los resultados originales de proyectos de investiga- ción científica y/o desarrollo tecnológico. Los procesos de los que se derivan están explícitamente señalados en el documento publicado, así como el nombre de sus autores y su afiliación institucional. La estructura generalmente utilizada consta de: introducción, metodología, resultados, conclusiones.

b. Artículos de reflexión: documentos que corresponden a resultados de estudios realizados por el o los autores sobre un problema teórico o práctico, que al igual que los anteriores, satisfacen las normas de certificación sobre la originalidad y calidad por árbitros anónimos calificados. Presenta resultados de investigación desde una perspectiva analítica, interpretativa o crítica del autor sobre un tema específico recurriendo a fuentes originales.

c. Artículos de revisión: estudios hechos por el o los autores con el fin de dar una perspectiva general del estado de un dominio específico de la ciencia y la tecnología, de sus evoluciones durante un período de tiempo y donde se señalan las perspectivas de su desarrollo y de evolución futura. Estos artículos son realizados por quienes han logrado tener una mirada de conjunto del dominio y están caracterizados por una amplia revisión bibliográfica de por lo menos 50 referencias.

d. Artículos cortos: documentos breves que presentan resultados originales preliminares o parciales de una investigación científica o tecnológica, que por lo general requieren de una pronta difusión.

e. Reportes de caso: documentos que presentan los resultados de un estudio sobre una situación particular, con el fin de dar a conocer las experiencias técnicas y metodológicas, consideradas en un caso específico. Incluye una revisión sistemática comentada de la literatura sobre casos ànálogos.

f. Revisión del tema: documento resultado de la revisión crítica de la literatura sobre un tema particular.

- Para dar cumplimiento a los requisitos de indexación, los artículos deberán ser sometidos a evaluación del Comité de Árbitros nacionales e internacionales, cuyo nivel de formación será de Maestría o Doctorado.

- Los artículos serán recibidos hasta el día 30 de mayo de cada año en las oficinas de la Especialización en Lenguaje y Pedagogía de Proyectos (Calle 34 No. 15-31 piso 4 Bogotá D.C.) telefax 3200771 o en el corréo electrónico plengua@udistrital.edu.co

- Una vez evaluados, se procederá a la selección de los artículos que serán publicados, momento en el cual se informará a los autores sobre posibles sugerencias para su ajuste y reformulación de aspectos editoriales. Aquellos que a juicio de los evaluadores requieran ajustes de fondo serán devueltos a los autores y sometidos a consideración del Comité Editorial para el siguiente número.

- Los documentos serán ubicados en alguna de las siguientes secciones, de acuerdo con la naturaleza del texto: Artículos, Reseñas, Autor invitado (para el caso de las traducciones). 